\title{
Identifying barriers to evidence-based practice adoption: A focus group study
}

\author{
Leilani Tacia ${ }^{* 1}$, Karen Biskupski ${ }^{1}$, Alfred Pheley ${ }^{1}$, Rebecca H. Lehto ${ }^{2}$ \\ ${ }^{1}$ Allegiance Health System, Jackson, MI, United States \\ ${ }^{2}$ Michigan State University College of Nursing, East Lansing, MI, United States
}

Received: January 3, 2015

Accepted: January 28, $2015 \quad$ Online Published: February 4, 2015

DOI: $10.5430 /$ cns.v3n2p90

URL: http://dx.doi.org/10.5430/cns.v3n2p90

\begin{abstract}
Objectives: The promotion of evidence-based practice (EBP) to ensure that the best scientific evidence, clinician expertise, and patient advocacy are used in health care delivery is an important leadership role of advanced practice nurses. While there has been much progress in advancing EBP, there are many hospital systems in the United States and other parts of the world that have yet to integrate an EBP framework. A focus group study was conducted to examine nurse's views relative to the adoption of EBP in a specific community hospital setting.

Methods: Design: A focus group design was used. Setting: A Midwestern United States rural community hospital. Sample: Four nurse practitioners, three nurse administrators/managers, and eleven inpatient direct care nurses. Three focus groups were conducted. Data were analyzed using qualitative methodology.

Results: Themes identified included institutional and/or cultural barriers, lack of knowledge, lack of motivation, time management, physician and patient factors, and limited access to up-to-date user-friendly technology and computer systems.

Conclusions: Engaging a participatory approach, findings provide strategy to consider when developing programs to expose nurses to EBP and for the translation of current standards into clinical practice. Building infrastructure to sustain and support EBP via leadership; time provision; access and support for continuing education; and collaborative integration of team members will guide the development of clinical environments that promote EBP.
\end{abstract}

Key Words: Clinical nurse specialist, Evidence-based practice, Community hospital

\section{Introduction}

Use of evidence-based practices ensures the best scientific evidence, clinician expertise, and patient advocacy are used in health care delivery. Promotion of evidence-based practice (EBP) is an important leadership role of clinical nurse specialists. $^{[1-3]}$ Practicing nurses must be prepared to formulate questions, critically assess practice, and evaluate research, clinical guidelines, and levels of evidence. ${ }^{[4]}$ Despite substantive development and systems in place to pro- mote EBP, many hospital systems internationally have yet to integrate an evidence-based model of care. ${ }^{[1,5]}$ Barriers to successful implementation arise from multiple factors including varying education and clinical experiences of nursing staff, and a lack of understanding about its' importance to optimal high quality patient care. ${ }^{[3,6,7]}$

Focus group research allows researchers to generate data that provides insight into the unique personal experiences and views of participants. ${ }^{[8-10]}$ Such data can be used by

*Correspondence: Leilani Tacia; Email: leilani.tacia@allegiancehealth.org; Address: Allegiance Health System, Jackson, MI, United States. 
clinical nurse specialists in the development of targeted strategies to respond to particular problems that challenge specific clinical settings. ${ }^{[8,9]}$ This focus group study examined nursing staff perceptions, attitudes, and beliefs regarding the adoption of EBP in a community hospital system recognized to be early in its transition to evidenced based care.

\subsection{Background and significance}

Wider adoption of EBP is yet needed to improve quality, safety, and promote effective care that can positively impact patient outcomes. ${ }^{[2,11-13]}$ Sparked by several Institute of Medicine reports published since 2001 focusing broadly on healthcare quality and redesigning systems to ensure patients receive safe, effective, patient centered, timely, efficient and equitable care, much progress has been made in developing systems to support EBP. ${ }^{[13]}$ However, only in the past decade has EBP been incorporated into nursing curricula, and many practicing nurses still lack knowledge about implementation. ${ }^{[3]}$ As a result, patients across the United States and other parts of the world may still receive care that is not based on current scientific evidence, including health care that may be unnecessary or have negative effects. ${ }^{[14]}$

While there have been advancements, translation of research into practice settings, incorporation of clinical practice guidelines to promote high quality care, and systematically changing institutional culture remain serious public health issues for some hospital systems. ${ }^{[5,15]}$ It is recognized that lag time from translating evidence to practice can take anywhere from eight to thirty years. ${ }^{[16,17]}$ Several well-accepted inpatient practice guidelines exist for pain, diabetes care, wound healing, and heart failure management, and have been incorporated into the medical record and into patient care. Patients experiencing these conditions, however, may still experience preventable adverse outcomes. $^{[2,11]}$

Previous reports of attitudes and knowledge of nurses towards EBP demonstrates a wide range of awareness, resources, and implementation. ${ }^{[17]}$ While nurses may be positive about EBP, this does not translate into implementation. ${ }^{[18,19]}$ Studies suggest that consistent educational programs may help build positive attitudes and knowledge, and nursing leadership is critical to promoting a positive EBP climate. ${ }^{[7,12,17,20]}$

The application of EBP can be challenging and requires collaboration and teamwork among nurses practicing in various roles. ${ }^{[13,19]}$ While organizational culture is critical to ensuring that a best practice climate is fostered, successful EBP requires more than positive leadership. ${ }^{[21]}$ At the foundation is a well-prepared workforce that moves the institution forward. ${ }^{[17]}$ Nurses in advanced roles are recognized as critical to leading a cultural shift that promotes EBP in the hospital environment and across the scope of health care inter- nationally. ${ }^{[7]}$ A recognized participatory approach to facilitate change and buy-in is to involve the stakeholders such as practicing nurses in the early development of a project. ${ }^{[19]}$ Therefore, the focus group methodology was considered a possible tool for better understanding the challenges of EBP implementation in a specific practice environment.

\subsection{Theoretical framework}

The Advancing Research and Clinical Practice through Close Collaboration (ARCC) Model was developed to improve integration of research and clinical practices in acute and community health care settings. ${ }^{[2]}$ The AARC model was conceptualized as a broad systematic strategy incorporating EBP mentorship, reliable information, and support to overcome institutional barriers. ${ }^{[2]}$ The model further emphasizes the importance of change. An important step to EBP adoption is to conduct an organizational assessment of implementation readiness for personal and institutional change. ${ }^{[23]}$

The purpose of the study was to examine nurses' perceptions, attitudes, and beliefs about EBP using focus groups. While limited clinical research has used focus group methodology, the established approach permits participants with shared background to discuss perspectives in a format where they can exchange meaningful information that leads to common themes. ${ }^{[8,24,25]}$ Study objectives included to: 1) gain an understanding of EBP perceptions based on practice environments and educational backgrounds; 2) explore specific barriers to EBP implementation unique to the specific cultural paradigm; and 3) elicit recommendations to enhance promotion of EBP. Such appraisal of nurse perceptions and experiences will be instrumental in guiding targeted strategies to promote best practice in the respective hospital culture. Further, the approach may be applicable to clinical nurse specialists promoting such progress in other unique practice settings.

\section{Methods}

The hospital Institutional Review Board approved all study procedures prior to the beginning of participant recruitment and study implementation.

\subsection{Setting and sample}

Three focus groups were conducted, coordinated by a site clinical nurse specialist with support from an academic partnership with a local university that has strong community involvement. The aim of the academic partnership was to assist with promotion and adoption of an evidenced-based practice culture and to foster nurse-led clinical research. Study participants were recruited from a community hospital located in the Mid-western region of the United States. Aiming to maximize variation in perspectives and views, included in the study were registered nursing staff with ad- 
vanced practice roles (such as nurse practitioners, clinical nurse specialists), administrative managerial roles, and inpatient direct care providers. Participants in each focus group coincided with the proportion of associated numbers of registered nursing personnel in the respective practice categories at the identified institution. Thus, there were fewer advanced practice nurses and management nurses who were included compared to numbers of nurses who provided direct bedside care. The three focus groups con- sisted of registered nurses in the following roles: advanced practice nurses (APN) $(n=4)$, nursing managers and/or administrators $(n=3)$, and inpatient direct care nurses $(n=$ 11). The APN's were masters' degree prepared, the administrator/managers group were bachelors' degree prepared, and 9 of the 11 inpatient direct care nurses (82\%) were associate degree prepared. Table 1 provides sample demographic data.

Table 1: Focus group nurse participant characteristics

\begin{tabular}{|c|c|c|c|}
\hline & Advanced practice nurses $(N=4)$ & Administrators/Managers $(\mathrm{N}=3)$ & Staff nurses $(\mathrm{N}=11)$ \\
\hline Age range (Median; Range) & $M=50 ; 37-62$ years & $\mathrm{M}=45 ; 27-57$ years & $M=36 ; 20-55$ years \\
\hline $\begin{array}{l}\text { Years of nursing experience (Mean } \\
\pm \text { Standard deviation; Range) }\end{array}$ & $26.75 \pm 10.69 ; 16-41$ years & $18.0 \pm 19.16 ; 5-40$ years & $6.45 \pm 5.57 ; 1-19$ years \\
\hline Sex N (\%) & Female = $3(75 \%)$ & Female = $3(100 \%)$ & Female = $9(82 \%)$ \\
\hline \multicolumn{4}{|l|}{ Work Environment N (\%) } \\
\hline Inpatient & $\mathrm{N}=0$ & $\mathrm{~N}=3(100 \%)$ & $\mathrm{N}=11(100 \%)$ \\
\hline Outpatient & $\mathrm{N}=3(75 \%)$ & $\mathrm{N}=0$ & $\mathrm{~N}=0$ \\
\hline Both & $\mathrm{N}=1(25 \%)$ & $\mathrm{N}=0$ & $\mathrm{~N}=0$ \\
\hline \multicolumn{4}{|l|}{ Shift Worked N (\%) } \\
\hline Days & $\mathrm{N}=3(75 \%)$ & $\mathrm{N}=3(100 \%)$ & $\mathrm{N}=6(55 \%)$ \\
\hline Nights & $\mathrm{N}=0$ & $\mathrm{~N}=0$ & $\mathrm{~N}=5(45 \%)$ \\
\hline Variable (Days/evenings) & $\mathrm{N}=1(25 \%)$ & $\mathrm{N}=0$ & $\mathrm{~N}=0$ \\
\hline \multicolumn{4}{|l|}{ Education Background N (\%) } \\
\hline Associates & $\mathrm{N}=0$ & $\mathrm{~N}=0$ & $\mathrm{~N}=9(82 \%)$ \\
\hline Bachelor of Science & $\mathrm{N}=0$ & $\mathrm{~N}=3(100 \%)$ & $\mathrm{N}=2(18 \%)$ \\
\hline Master of Science & $\mathrm{N}=4(100 \%)$ & $\mathrm{N}=0$ & $\mathrm{~N}=0$ \\
\hline
\end{tabular}

\subsection{Data collection}

Nurses were recruited for specific groups based on job title and work roles via email and flyer distribution. Established focus group rules included the voluntary nature of participation and use of audio recording. In addition, group conduct rules including the importance of strict confidentiality, respect for alternative viewpoints, the need to ensure that all participants had the chance to share perspectives, and use of first names only during discussions were incorporated. Each focus group was conducted in the same conference room and informed consent was obtained from all participants. Each focus group was completed within a two-hour time frame. Refreshments were provided and participants were free to leave early, as needed. A doctoral prepared nurse scientist familiar with focus group methodology who was aligned with the academic partnership institution moderated each session using the pre-established questions and probes (see Table 2). Also participating in focus group coordination and conduct were the site principal investigators, a master's degree prepared clinical nurse specialist and a bachelor's prepared nurse manager. While the nurse scientist who moderated the focus groups did not know the participants, the site principal investigators had previous personal interactions with some participants working in the same organization. It is understood in qualitative methodology that researchers bring inherent bias based on their personal viewpoints and background into the study dynamic. In this line, site investigators worked to facilitate a nonthreatening supportive atmosphere and reflective dialogue among the focus group participants while avoiding interjecting personal opinions and interpretation. 
Table 2: Examples of focus group questions and sub-probes

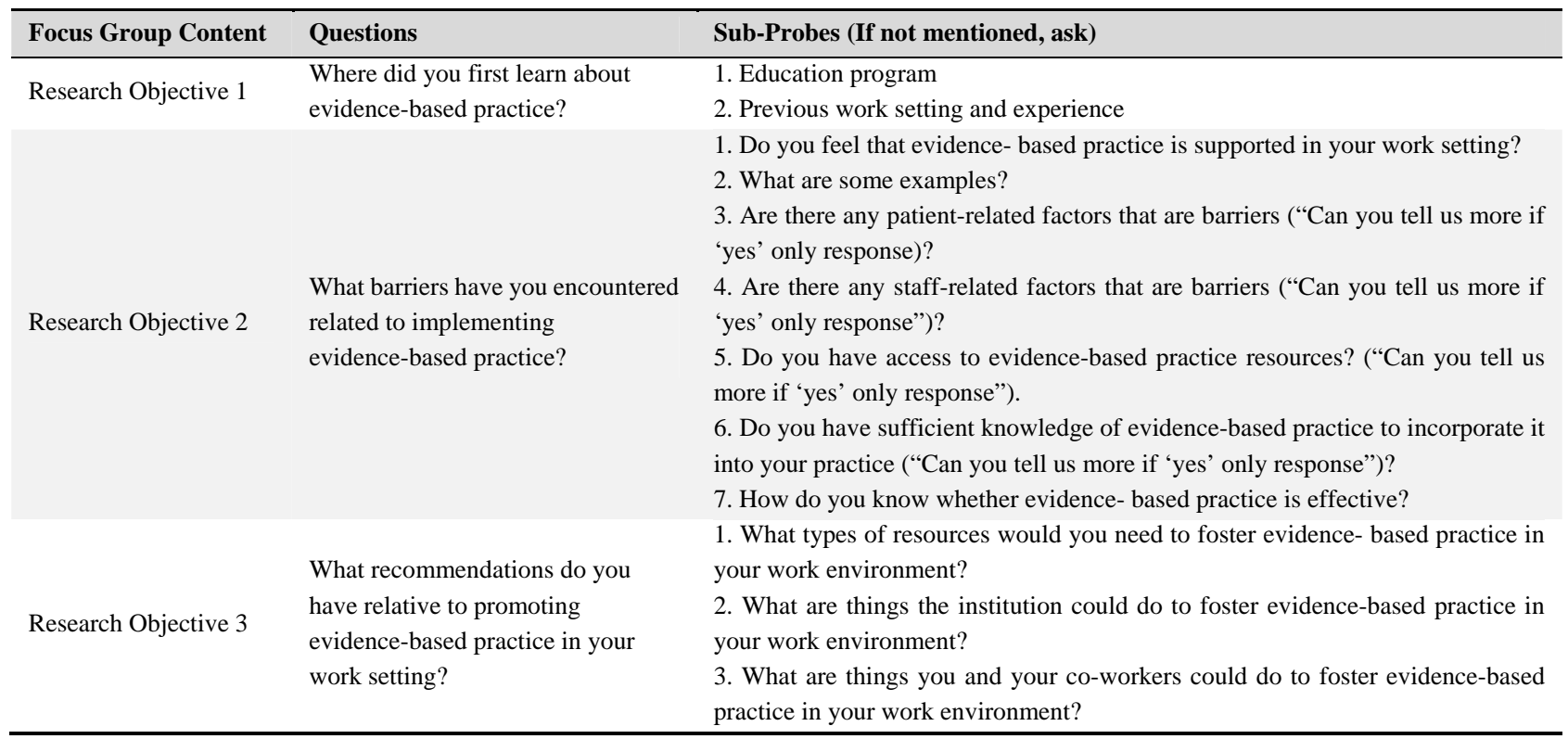

\subsection{Data analysis}

Using procedures established by Krueger and Casey ${ }^{[8,25]}$ the tapes were transcribed verbatim. Transcriptions were reviewed for accuracy and independently examined by the investigators. Analysis was applied towards the recognition and modification of major themes and sub-themes. ${ }^{[8,24,25]}$ Data were compared across the three focus group encounters with focused attention to group similarities and differences. Content categories were developed, and agreement related to content was obtained following the two independent reviews and follow-up discussions among study investigators.

\section{Results}

Six factors were identified as challenges to EBP adoption: institutional and/or cultural barriers, lack of knowledge, lack of motivation, time management, physician and patient factors, and limited access to up to date, user-friendly technology and computer systems. Nurses also discussed strategies to improve adoption of EBP. Specific examples of the major themes are provided in the following paragraphs.

\subsection{Institutional and/or cultural barriers}

Members of the three groups identified cultural factors within the institution and the need to promote system-wide hospital change as being significant barriers. One nurse practitioner described the culture as being one that did not demand best practice from the nurses. "I think in places that back up their practice with evidence, there's this culture of learning and of 'we want it to be the most current and up to date'. That's when you hear guys walking around hallways and quoting [EBP from] studies, and we don't have that here so there has to be a culture change."

An inpatient direct care nurse described that the importance of professional advancement among nurses was undermined because of cultural attitudes of the hospital system. "I ... wanted my specialty in oncology and my preceptor said, why? You're not going to get any more money ... You know [named hospital] doesn't care if you've got that. I said I'm doing it for me. I want to explain to my patient why the OCN [oncology certified nurse] is behind my name."

Nurse practitioners discussed traditional norms and a strong tendency to fall back on habits rather then base practice on current evidence. One nurse practitioner stated, "When you bring it [EBP] up to the nurses, if you ask them why you do it this way... They say it's because we've always done it. When you look at the whole, it's more of a process issue ... because that's how we've always done it than an actual task itself." Another nurse practitioner stated, "There are some things we do that we blatantly say, 'there is no evidence for this, we're just going to do it.' Some of that is because 'guys' are a little stodgy and stuck in their way and its' always worked this way before, so it's obviously good practice. It's the way we've always done it, and there's no clear evidence to contradict this and there is a comfort level there in doing this, so that actually inhibits in varying degrees our ability to tackle this ..."

\subsection{Lack of knowledge}

Nurse practitioners and nurse managers felt that many inpatient direct care nurses did not have a basic knowledge of EBP. Further, these nurses may not be able to recognize criteria that reflect high quality research. "I think it's a challenge ... to really grasp where to look because it's so easy to Google something. But to know what is truly supported by 
good research and to know the difference rather than it's Dr. Oz saying it ... To know it's from a good nursing or medical journal." Nurse practitioners described inpatient direct care nurses who were not adequately prepared. "They're very task focused. ... and then recently they did the whole nurse survey of education levels, and so I saw that and I thought ohhh ... there are so few BSN [bachelor of science in nursing] nurses here." Importantly "lack of knowledge" was not discussed as a theme by the inpatient direct care nurses in their respective focus group.

\subsection{Lack of motivation}

Inpatient direct care nurses described the importance of having personal drive and motivation to improve themselves to provide better professional care. In some discussions, nurses verbalized not being interested or inspired to adopt EBP. In one specific example, a inpatient direct care nurse stated: "Seeing that a lot of nurses have been here a long time which is a really good thing that there is so much longevity... But it is easy when you are entrenched... having spent many years at one facility, you become stagnant, that is a good term for it." Managers described nurses as not motivated to implement EBP because of the length of time between formal academic training and current employment. "A lot of them ... want to come and do their job and... Somewhere along the line ... they have forgotten about the need to stay current, their responsibility to the profession, their patients, and to their license..."

\subsection{Time management}

Time commitment was a barrier to implementation of system-wide changes identified in all three focus groups. One nurse practitioner expressed concern that it was not realistic to expect nurses to follow up with research to back up their practice. "They are trying to take care of patients ... They're not gonna (sic) look up an article ... [They] would love to look this stuff up and understand it more, but they don't have the time or the options, and then they're off hours." A nurse manager stated: "Implementing evidencebased practice is very time consuming, and there are a lot of hoops and bells to jump through and people to talk to to get anything implemented, so that's a barrier. Where you have nurses or even mid-level staff bringing in really good research-based evidence and to get it presented to the doctors and to get everyone on board, and then do the education so that everyone knows it... I think that's a bigger obstacle than just getting to the research. It's what do I do with it once I get it, and what's the steps and a timely manner and have a response one way or another..."

\subsection{Physician and patient factors}

Nurse practitioners noted that both doctors and patients had different values and beliefs that may conflict with providing care based on high evidence. One nurse practitioner de- scribed doctors who did not question their traditional practice habits. "It's a challenge because the doctors practice a certain way and not that they don't care, but they feel their way is evidence based even though it may be outdated. So that's the challenge... that the doctors want to keep their evidence base even though it may be from 10 years ago."

Nurse practitioners felt that patients did not want to change their treatment plans when new evidence emerged. An example of one discussion was: "If you bring something new to the table to them [older patients], it is quite a struggle to get them convinced to change... 'I've been on this beta blocker for 30 years. I've always been on Lasix and that's what they use to control my blood pressure'. 'But you're frail now and you shouldn't have it'. So trying to convince them ... or their family who their loved one has been on Aricept [Donepezil] or Nemanda [Memantin] for 10 years and now tell them it's not any good any more, research is showing us that ... and they say ... please don't take it away, I don't care what your research says."

\subsection{Limited access to up-to-date user-friendly tech- nology and computer systems}

Adequate computer resources were essential for EBP implementation as identified by a nurse practitioner and nurse manager. A nurse manager stated: "Your computer systems .... [are] very lacking and challenging to work with. [It] was phenomenally hard to navigate." Nurse practitioners' identified that inpatient nurses who provide direct patient care require access to and assistance with technical resources.

\subsection{Strategies to improve adoption of evidence- based practice}

Nurses' expressed interdisciplinary communication and collaboration, mentorship, access to professional activities and networks, and supportive management as crucial to EBP adoption. Inpatient direct care providers emphasized that management needed to champion advanced education for bedside nurses.

One nurse practitioner stated that physician support was needed to make the transition to evidence-based care. "The physician may see it [evidence read in the literature] as a threat to their power or knowledge base. How many times have you heard 'you think you know more than I do?'” A nurse practitioner stated: "I see it when you go to critical care. They [the nurses] have a lot more autonomy because of their comfort with their relationship with the doctor. More than anything, the skill is there in general, but it's what they've been empowered to do. You have strong nurses in the specialty setting, and they're comfortable with what they know."

Participants described ways to inspire EBP adoption. Conference attendance, training sessions, and organizational 
support for education (conference reimbursement, incentives) were viewed as inspirational. "Trying to bring back information when you go to conference that's different, that people are doing in other places ... That exposure and to share that information I think is important ... I mean you can read it in your journals, but it's a lot more interesting when you meet the people whose names are behind the article in the journal."

\section{Discussion}

While EBP has become an integral component of optimal patient care, nursing professionals practicing in many hospital systems globally continue to experience barriers to promotion of evidence-based care. In this study, focus group discussions were used to elicit perspectives regarding professional and institutional barriers related to EBP adoption. In line with other studies, ${ }^{[18,20,26,27]}$ participants identified limited resources as a primary concern to EBP implementation. Specific barriers included time, access to quality information, knowledge to translate evidence to practice, and interdisciplinary collaboration. Task-based care may divert nurses' attention away from questioning traditional practices considering best practice alternatives. ${ }^{[7,12]}$

Professional development to gain EBP knowledge is recognized as the nurse's best preparation for providing clinical care that optimizes patient outcomes. ${ }^{[28]}$ There may be less incentive to aspire professionally in cultures where there is a perception that there will not be managerial support and recognition. ${ }^{[4,7,18]}$ Similar to other studies, opportunities and institutional support to attend conferences, further education, and gain advanced training were viewed as important factors to inspire EBP adoption. ${ }^{[27,29]}$

Nurses' in the focus groups identified that medical doctors can be resistant to change. Collaborative relationships with inter-disciplinary staff are integral to ensure that the patients receive care based on the best available evidence. ${ }^{[16]}$ Strategic context-sensitive initiatives are needed to ensure that all team members (inpatient direct care nurses, doctors, management, nurses in advanced roles, etc.) recognize a common mission and their accountability in ensuring that patients receive care based on best evidence. Although challenging, team-oriented methods to enhance communication and to promote strong relationships among staff are essential to lead organizational change. Early foundations can be laid with a motivated nurse research council to familiarize nurses with clinical research and the benefits of EBP. ${ }^{[17,28]}$ Evidence-based fellowships that attract motivated clinical nurses to lead change and inspire practice settings to ask questions and be resourceful are increasingly seen as an essential tool to motivate change. ${ }^{[28]}$ With trained mentors familiarized with the cultural setting, such innovative programs can serve as a bridge to bring real-world research to the bedside to improve patient care quality. ${ }^{[28]}$ In environ- ments where the majority of inpatient direct care nurses are associate degree prepared, there is an increased need for motivated clinical nurses to provide guidance and mentorship to their colleagues.

The focus group approach limits the nurse researcher to small convenience samples of participants for institutional representation. Along this vein, the nurses who participated in the focus groups may have consisted of those inspired to make practice changes or who wanted to express disgruntlement. Further, given most $(82 \%)$ of the nurses providing inpatient direct care who participated were associate degree prepared nurses', they may have lacked formal EBP educational preparation. It is recognized that focus group formats may limit discussion if there are informal leaders among participants. ${ }^{[8,10]}$ For example, alternate perspectives may not emerge if members were cautious of raising contrary opinions. Focus group findings are not meant to be transferable to other populations or situations, but identify unique themes addressable to the specific cultural setting. ${ }^{[5,10]} \mathrm{Im}$ portantly, clinical nurse specialists may find the focus group format helpful to gain valuable information about barriers in their respective work sites, while promoting a participatory approach that makes nurses feel that their perspectives are valuable. Limitations of focus group methodology include that groups require participant trust, information can be withheld and/or be aligned with what the participants think the facilitator wants to hear, and data may be limited by "group think" that confines the discourse to one perspective. ${ }^{[10]}$ Another limitation of this study is that only one focus group was conducted with each of the respective groups of nurses. It is plausible that more information could have been gleaned by conducting further focus groups with different groups of participants. Thus, future studies of this nature should involve more sessions to ensure that all the essential information, or data saturation, is attained.

\section{Conclusions}

The focus group findings provide important context-specific information relative to staff readiness to adopt EBP. While the presence of pro-active nurse practitioners who have training and familiarity with EBP and are strengths, these advanced practice nurses tended to be negative in their attitudes about the potential of staff nurses to move forward with a practice guided by evidence. Importantly, however, nurses who with advanced training have the clinical skills and experience to partner in promoting and facilitating EBP for clinical nurses. ${ }^{[15,27]}$ It is recognized that hospital culture that stimulates ongoing inquiry and utilization of the best available evidence guides care towards optimizing patient outcomes. Building infrastructure to sustain and support EBP via leadership; the provision of time; access to and support for continuing education; and collaborative integration of team members will guide the development of clinical environments that promote EBP at the patient level. Clini- 
cal nurse specialists who are at the forefront in setting the stage for strategic initiation of professional organizational shifts to promote best care may find the focus group approach helpful in guiding such efforts.

\section{Acknowledgements}

The authors would like to acknowledge Allegiance Health
System and the nurse participants for their time, support, and commitment.

\section{Conflicts of Interest Disclosure}

There are no conflicts of interest or funding sources to report.

\section{References}

[1] Austin L, Luker K, Martin R. Clinical nurse specialists and the practice of community nurses. J Adv Nurs. 2006; 54: 542-552. http: //dx.doi.org/10.1111/j.1365-2648.2006.03868_1.x

[2] Heitkemper MM, Bond E. Clinical nurse specialist: State of the profession and challenges ahead. Clin Nurs Spec. 2004; 18: 135-140. http://dx.doi.org/10.1097/00002800-200405000-00014

[3] Linton MJ, Prasun MA. Evidence-based practice: Collaboration between education and nursing management. J Nurs Manag. 2013; 21 : 5-16. http://dx.doi.org/10.1111/j.1365-2834.2012.014 $40 . \mathrm{x}$

[4] Koehn ML, Lehman K. Nurses' perceptions of evidence-based nursing practice. J Adv Nurs. 2008; 62: 209-215. http://dx.doi.org /10.1111/j.1365-2648.2007.04589.x

[5] Calarco MM. The impact of positive practices on nurse work environments: Emerging applications of positive organizational scholarship. West J Nurs Res. 2011; 33: 365-384. http://dx . doi . org/1 $0.1177 / 0193945910380211$

[6] Atkinson M, Turkel M. Overcoming barriers to research in a magnet community hospital. J Nurs Care Qual. 2008; 23: 362-368. http: //dx.doi.org/10.1097/01.NCQ.0000336675.48466.37

[7] Fineout-Overholt E, Levin RF, Melnyk B. Strategies for advancing evidence-based practice in clinical settings. J New York Nurs Assn. 2004/2005; 28-32.

[8] Krueger A, Casey MA. Focus Groups: A Practical Guide for Applied Research. 3rd ed. London, England: Sage; 2000.

[9] Gibson F, Bamford O. Focus group interviews to examine the role and development of the clinical nurse specialist. J Nurs Management. 2001; 9: 331-342. http://dx.doi.org/10.1046/j.096 6-0429.2001.00267.x

[10] Marrelli AF. Collecting data through focus groups. Perform Improve. 2013; 47: 39-45. http://dx.doi.org/10.1002/pfi. 201

[11] Taylor R. Using and developing the evidence base in primary health care. Prim Health Care. 2012; 22: 31-36. http://dx.doi .org/1 $0.7748 / \mathrm{phc} 2012.02 .22 .1 .31 . \mathrm{c} 8916$

[12] Fineout-Overholt E, Melnyk B. Building a culture of best practice. Nurs Leader. 2005; 26-29. http://dx.doi.org/10.1016/j .mnl .2005.09.007

[13] Stevens K. The Impact of Evidence-Based Practice in Nursing and the Next Big Ideas. OJIN: The Online Journal of Issues in Nursing. 2013; 18(2): Manuscript 4. http://dx.doi.org/10.3912/0JI N. Vol18No02Man04

[14] Larkin M, Cierpial C, Vanderboom T, et al. Research ambassadors: Bringing findings to the bedside. Nurs Manag. 2009; 40: 20-23. http://dx.doi.org/10.1097/01. NUMA.0000361805. 74369.23

[15] Gerrish K, Nolan M, McDonnell A, et al. Factors influencing advanced practice nurses' ability to promote evidence-based practice among frontline nurses. Worldviews Evid-Based Nurs. 2012; 9: 3039. http://dx.doi.org/10.1111/j.1741-6787.2011.00230 $\cdot \mathrm{x}$
[16] Everett LQ, Siterding MC. Transformational leadership required to design and sustain evidence-based practice: A system exemplar. West J Nurs Res. 2011; 33: 398-426. http://dx.doi.org/10. 1177/0193945910383056

[17] Sandström B, Borglin G, Nilsson R, et al. Promoting the implementation of evidence-based practice: A literature review focusing on the role of nursing leadership. Worldviews Evid-based Nurs. 2011; 8: 212-223. http://dx.doi.org/10.1111/j.1741-678 7.2011.00216.x

[18] Stokke K, Olesen N R, Espehaug B, et al. Evidence based practice beliefs and implementation among nurses: A cross-sectional study. BMC Nurs. 2014; 13: 1-10. http://dx.doi.org/10.1186/147 2-6955-13-8

[19] Brewer B, Brewer M, Schultz A. A collaborative approach to building the capacity for research and evidence-based practice in community hospitals. Nurs Clin North Amer. 2009; 44: 11-25. http: //dx.doi.org/10.1016/j.cnur.2008.10.003

[20] Nagy S, Lumby J, McKinley S, et al. Nurses' beliefs about the conditions that hinder or support evidence-based nursing. Int $\mathbf{J}$ Nurs Pract. 2001; 7: 314-321. http://dx.doi.org/10.1046/j.144 0-172X.2001.00284.x

[21] Rickbeil P, Simones J. Overcoming barriers to implementing evidence-based practice. J Nurs Staff Dev. 2012; 28: 51-56. http: //dx.doi.org/10.1097/NND.0b013e31824b4141

[22] Melnyk BM, Fineout-Overholt E. Putting research into practice. Rochester ARCC. Reflect Nurs Lead. 2002; 28: 22-25. PMid:12073802.

[23] Melnyk BM, Fineout-Overholt E. The Evidence-Based Practice Beliefs and Implementation Scales: Psychometric Properties of Two New Instruments. Worldviews Evid-based Nurs. 2007; 4: 208-215. http://dx.doi.org/10.1111/j.1741-6787.2008.00126.x

[24] Barbour RS. Making sense of focus groups. Med Educ. 2005; 39: 742-750. http://dx.doi.org/10.1111/j.1365-2929.20 $05.02200 . \mathrm{x}$

[25] Curtis EA, Redmond R. Focus groups in nursing research. Nurs Researcher. 2007; 14: 25-37. http://dx. doi.org/10.7748/nr200 7.01.14.2.25.c6019

[26] Gawlinski A, Rutledge D. Selecting a model for evidence-based practice changes: A practical approach. AACN Adv Crit Care. 2008; 19: 291-300. http://dx.doi.org/10.1097/01. AACN.0 000330380.41766 .63

[27] Solomons NM, Spross JA. Evidence-based practice barriers and facilitators from a continuous quality improvement perspective: an integrative review. J Nurs Manag. 2011; 19: 109-120. http://dx .doi.org/10.1111/j.1365-2834.2010.01144.x

[28] Latimer R, Kimball J. Nursing research fellowship: Building nursing research infrastructure in a hospital. J Nurs Admin. 2010; 40: 92-98. http://dx.doi.org/10.1097/NNA.0b013e3181c b9f $9 e$

[29] Pierson M, Schuelke S. Strengthening the use of evidence-based practice: development of an independent study packet. J Cont Educ Nurs. 2009; 40: 171-176. http://dx.doi.org/10.3928/00220 124-20090401-09 